\title{
LOFAR - a new low-frequency radio telescope
}

\author{
Huub Röttgering ${ }^{1}$, Michiel van Haarlem ${ }^{2}$, and George Miley ${ }^{1}$ \\ ${ }^{1}$ Leiden Observatory, P.O. Box 9513, 2300 RA Leiden, The Netherlands \\ ${ }^{2}$ ASTRON, P.O. Box 2, 7990 AA Dwingeloo, The Netherlands
}

\begin{abstract}
LOFAR, the Low Frequency Array, is a new low frequency telescope that is currently being built in the Netherlands. It will initially observe at frequencies from 30 to $240 \mathrm{MHz}$ with baselines of up to $100 \mathrm{~km}$. The aim for the second phase is to carry out sensitive observations below $30 \mathrm{MHz}$ and to extend the maximum baselines up to $400 \mathrm{~km}$. The design of LOFAR is driven by four fundamental astrophysical applications: (i) the epoch of reionisation; (ii) extragalactic surveys and their exploitation to study the formation and evolution of clusters, galaxies, and black holes; (iii) transient sources and their association with high energy objects such as gamma ray bursts; and (iv) cosmic ray showers and their exploitation to study the origin of ultra-high energy cosmic rays. Particularly relevant for this conference is LOFAR's ability to study the epoch of reionisation through observations of the redshifted $21 \mathrm{~cm}$ line. This will address several important issues including the source of reionisation and the reionisation history of the Universe. In addition to the science case, LOFAR's instrumental concepts and time line are briefly reviewed. The main technical challenges for LOFAR include rejection of manmade radio interference, dealing with the corrupting influence of the ionosphere on the observations, and minimising contaminations of the EOR signal by foreground discrete astrophysical sources.
\end{abstract}

\section{Introduction}

LOFAR, the Low Frequency Array, is a next-generation radio telescope of which the main part will be located in The Netherlands. It will initially operate at frequencies from 30 to $240 \mathrm{MHz}$ (corresponding to wavelengths of 10 to $1.2 \mathrm{~m}$ ). LOFAR will facilitate a broad range of fundamental astrophysical studies by providing enormous improvements over previous facilities in the following three regions of parameter space:

- Very low frequencies, with $2-3$ orders of magnitude improvement in sensitivity attained at frequencies below $\sim 40 \mathrm{MHz}$. This is a relatively unexplored spectral region that is uniquely sensitive to coherent radiation mechanisms and the oldest fossil synchrotron electrons.

- Size of the instantaneous field of view, of thousands of square degrees which will enable searches for new classes of rare transient and variable sources, and studies of high energy cosmic rays through cosmic air showers. LOFAR will be the first synoptic radio telescope.

- Low-frequency radio spectroscopy, enabling studies of redshifted neutral hydrogen at the epoch of reionisation, one of the topics of this conference.

In this contribution we will briefly discuss: (i) LOFAR's instrumental concept and the status of the LOFAR project, (ii) the general astrophysical science case, and (iii) LOFAR's potential contribution for studying the epoch of reionisation. 
Table 1. Phase $1^{1,2}$ LOFAR specifications

\begin{tabular}{lll}
\hline Configuration & 45 remote stations and \\
& 32 stations in $2 \mathrm{~km}$ core \\
Longest baselines & $100 \mathrm{~km}$ & \\
Frequency range & $30-80 \mathrm{MHz}$ & $110-240 \mathrm{MHz}$ \\
Low-band antennas & 7700 & \\
High-band 4x4 antenna tiles & & 7700 \\
Collecting area & $10^{5} \mathrm{~m}^{2}$ at $50 \mathrm{MHz}$ & $10^{5} \mathrm{~m}^{2}$ at $150 \mathrm{MHz}$ \\
Angular resolution & $12^{\prime \prime}(50 \mathrm{MHz})$ & $4^{\prime \prime}(150 \mathrm{MHz})$ \\
Field of view (8 beams) & $\sim 325 \mathrm{deg}^{2}(50 \mathrm{MHz})$ & $\sim 35 \mathrm{deg}^{2}(150 \mathrm{MHz})$ \\
Field of view (all-sky mode, core $)$ & $30-80 \mathrm{MHz} 8100 \mathrm{deg}^{2}$ & $110-240 \mathrm{MHz}: 530 \mathrm{deg}^{2}$ \\
Sensitivity (4 h, $4 \mathrm{MHz})$ & $1 \mathrm{mJy}(50 \mathrm{MHz})$ & $0.05 \mathrm{mJy}(150 \mathrm{MHz})$ \\
\hline
\end{tabular}

${ }^{1}$ For Phase 2, the goal is to extend the frequency range down to $\sim 10 \mathrm{MHz}$ and the baseline lengths to $\sim 400 \mathrm{~km}$.

2 More information on the LOFAR specification can be found at http://www.lofar.org.

\section{The LOFAR telescope}

A summary of LOFAR's specifications is given in Table 1. LOFAR will have two antenna systems, one optimised for the $30-80 \mathrm{MHz}$ range, and one for the $110-$ $240 \mathrm{MHz}$ range. The low frequency antennas will be active crossed short dipoles. The high frequency antenna will be a $4 \times 4$ crossed dipole array, with integrated beam-former. A picture of a prototype antenna for the low frequency range is given in Fig. 1 . The antennas will be placed in soccer-field sized stations yielding, for each station, effective apertures that will range from $50 \mathrm{~m}$ to $150 \mathrm{~m}$, depending on frequency. A first LOFAR test station is operational; for a picture see Fig. 1. Albeit at the low angular resolution of 3 degrees, this station has produced a complete map of the northern sky with the Galactic plane and the brighter $3 \mathrm{C}$ radio sources clearly visible. It further has observed flares from the Sun and Jupiter and detected a number of high-energy cosmic rays.

The signals from each low frequency antenna and/or from each $4 \times 4 \mathrm{HF}$ array are digitised and fed into the station beam-former. The beam-former can produce up to eight coherent "station-beams" within the primary power pattern of the antenna element in use. The output data stream from each station beam is sent to an optical link for transmission to the central processing facility. The main computer at this facility, named "Stella', is an IBM bluegene/L computer, consisting of 6 racks, each with 1024 nodes. The theoretical maximum performance is 34 Tflops; a realistic maximum based on Top500 benchmarks is 26 Tflops.

The initial array will comprise 45 stations. These stations will be distributed over an area of diameter of $\sim 100 \mathrm{~km}$ (see Fig. 2), yielding an angular resolution of 3 arc-seconds at $200 \mathrm{MHz}$. The layout is designed to yield good instantaneous UV-coverage, and will be elongated in the N-S direction to produce roughly circular synthesised beams over a wide range of declinations. This will ensure significant overlap in accessible sky between LOFAR and southern hemisphere facilities such as the VLT and ALMA.

The aim for LOFAR's second stage is to have baselines extending up to at least $400 \mathrm{~km}$. Discussions with astronomers from European countries have started to build such an extended LOFAR. At the higher frequencies the resulting angular resolution will be better than 1 arc-sec and thereby well matched to optical, (sub-) mm, and X-ray observing facilities.

LOFAR's time line is ambitious. The critical design review is planned for Autumn 2005 , with the first operation of the central core toward the end of 2007. Baselines of $100 \mathrm{~km}$ should be available at the end of 2008 . 

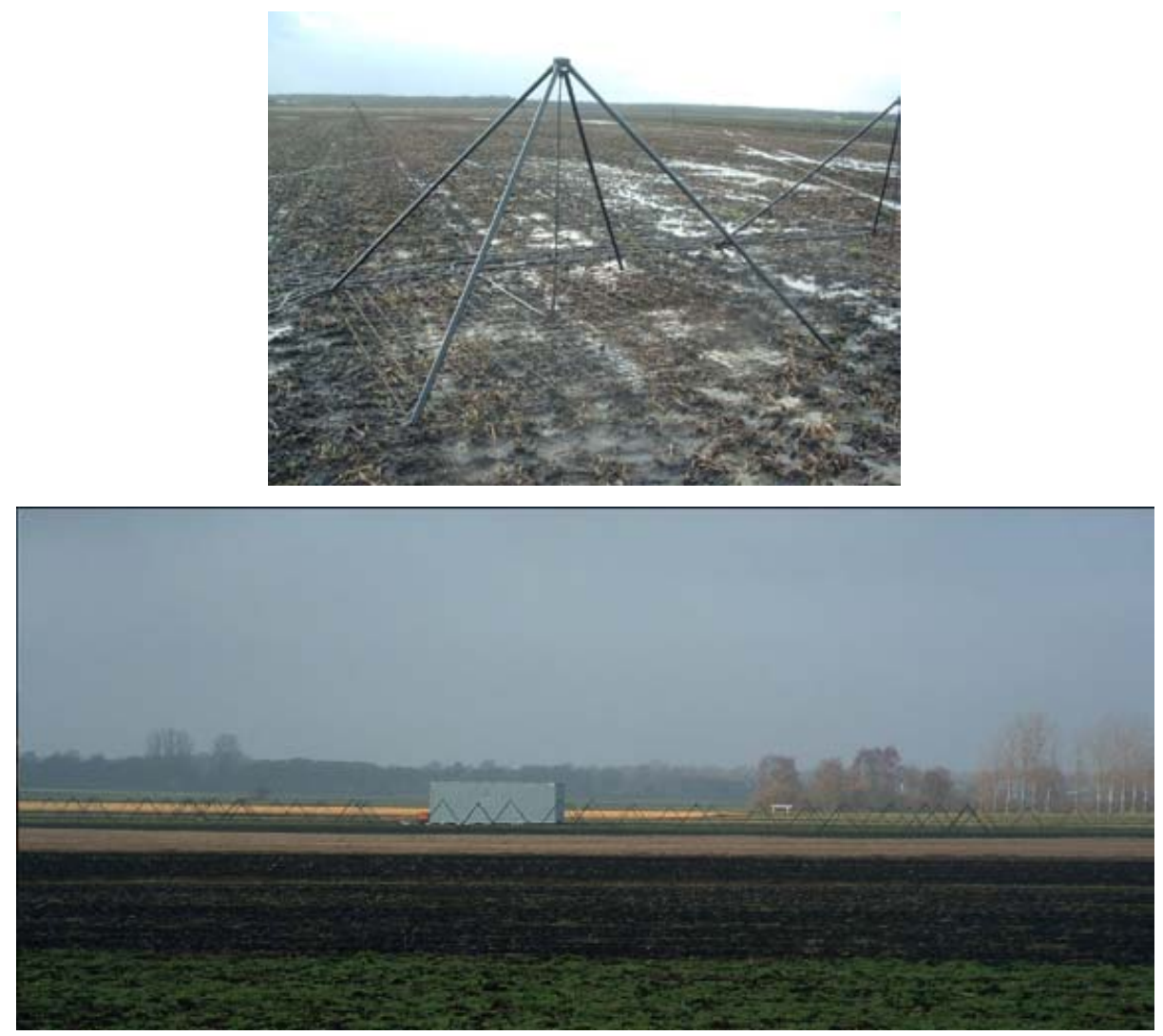

Figure 1. (upper) An engineering prototype of the Low Frequency Antenna. (lower) The LOFAR initial test station consisting of 64 antennas located near the municipality of Borger-Odoorn, in the Dutch province of Drenthe.

\section{LOFAR Scientific Objectives}

The sensitivities and spatial resolutions attainable with LOFAR will make possible several fundamental new studies of the Universe. These studies include:

- In the very distant Universe $(7<z<10)$, LOFAR can search for the signature produced by the reionisation of neutral hydrogen. Reionisation is one of the topics of this conference and the usage of LOFAR to study the epoch of reionisation will be discussed further in this contribution.

- In the distant formative Universe $(1.5<z<7)$, LOFAR will detect many millions of star-forming galaxies, very distant radio galaxies and quasars, and many thousands of diffuse radio clusters sources. These will be used to study the processes by which the earliest structures in the Universe (galaxies, clusters, and active nuclei) form, and probe the intergalactic gas.

- In the nearby Universe, LOFAR will map the 3-dimensional distribution of cosmic rays and global magnetic field in our own and nearby galaxies.

- LOFAR will detect flashes of low-frequency radiation from pulsars and short-lived transient events produced by stellar merging and interaction, and will search for Jupiterlike extra-Solar planets.

- Within our Solar system, LOFAR will detect coronal mass ejections from the Sun and provide continuous large scale maps of the Solar wind. 


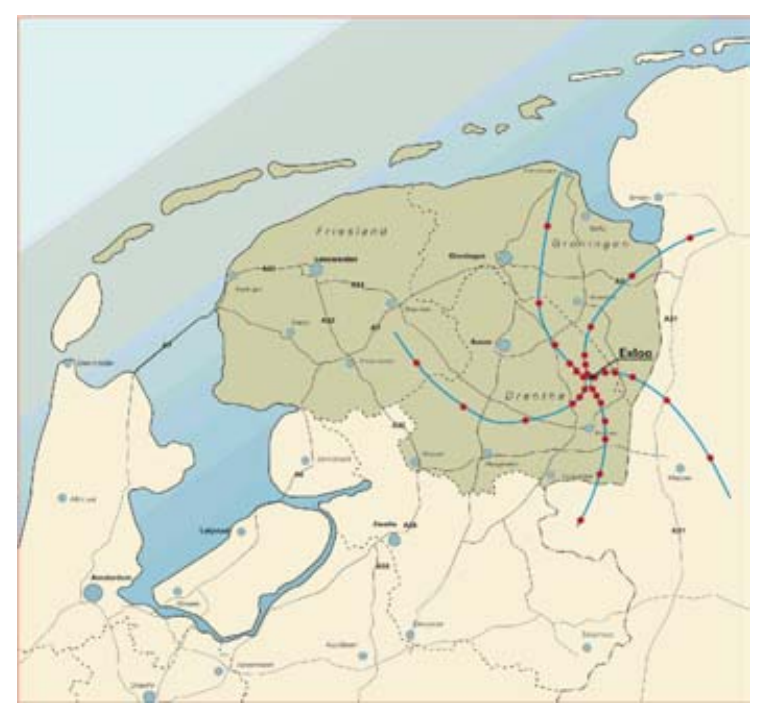

Figure 2. Approximate layout of the LOFAR stations in a 5-armed log-spiral pattern with North-South elongation in order to facilitate imaging at low declinations.

- LOFAR will also detect showers from ultra high energy cosmic rays as they pierce the Earth's atmosphere.

Furthermore, by exploring a new spectral window and by observing with an unprecedented field of view LOFAR is likely to make unexpected "serendipitous" discoveries. Detection of new classes of objects and/or new astrophysical phenomena have resulted from almost all previous facilities that opened new regions of the spectrum, or pushed instrumental parameters, such as sensitivity by more than an order of magnitude (e.g. Harwit 1981).

Finally, the LOFAR array will be used for a wide range of scientific applications not directly related to astronomy. Application areas include those of geophysics, ionospheric studies, and information technology.

\section{Reionisation}

One of the most exciting goals of LOFAR will be to chart the end of the Dark Ages when the neutral baryonic gas is being ionised. The tail of this transition from a neutral to an ionised Universe has been identified at a redshift $\sim 6-7$ by means of the GunnPeterson trough in the spectra of high redshift quasars in the SDSS survey (Fan et al. 2003; White et al. 2003). This revealed small amounts of neutral hydrogen at $z \sim 6$. The large-angle CMB polarisation signal measured by the Wilkinson MAP satellite indicates that the very first isolated stars have even commenced this reionisation of the Universe at the early stage of $z \approx 17$ (Kogut et al. 2003). These very different observational constrains suggest that reionisation might be taking place during an extended period with a number of competing ionisation mechanisms (e.g. Cen 2003).

The design of LOFAR's experiment to study the epoch of reionisation, the observations, calibration, and reduction methods will be presented in detail by de Bruyn et al. (2005). Here we briefly review and discuss the main issues. The main data product from LOFAR's EOR observations will be a 3-dimensional data cube with right ascension and declination as two axes and frequency on the third axis. The plan is to observe in the first years of 
operation of order 5 regions on the sky each covering an area of 100 sq. degrees. To reach the required depth of a few $\mathrm{mK}$ rms brightness temperatures, integration times of 50-100 nights will be required.

For the production of the such maps, three major technical challenges are foreseen (see also for example Kanekar \& Briggs 2004 and de Bruyn et al. 2005).

First, there is the issue of the measurements being corrupted by man-made radio frequency interference (RFI). This is dealt with in several ways. LOFAR will make use of high dynamic range 12 bit A/D converters, instead of the traditionally used 1-2 bits. This ensures that the systems remain perfectly linear in most of the frequency channels that are affected by interference. Furthermore, the spectral width of the interference is mostly very narrow band $(<1 \mathrm{kHz})$, thereby confining significant interference to less than a few percent of the frequency channels in the relevant receiver bands. One of the aims of the new WSRT Low Frequency Front End (LFFE) system, commissioned in Autumn 2004, was to test whether at high spectral resolution the impact of RFI on the LOFAR data would be acceptable. The conclusion from the test observations was that at an LFFE resolution of $9 \mathrm{kHz}$, often only $10-20 \%$ of the visibilities are affected. As already mentioned, LOFAR will observe at a resolution of $1 \mathrm{kHz}$, reducing channels affected by interference to an even smaller fraction. In addition, faint sources of RFI are not located at a constant position on the sky, and their signals will not add up coherently. These RFI sources will therefore represent a negligible noise term as compared to the high brightness temperature of the sky.

Second, there is the corrupting influence of the ionosphere on the data. The main way of dealing with this is to have sufficient collecting area per station that the behaviour of the ionosphere can be monitored and calibrated. The implementation of the calibration routines will rely on a generalised version of the self-calibration algorithms that have been successfully employed in traditional interferometers for many years now (Noordman 2004).

Third, the final technical challenge will be the large amount of storage and computing facilities needed to properly reduce the data. A full data set obtained after a few months of observing will contain at least a petabyte of data. Estimates indicate that the computer resources available to the project are sufficient to coherently calibrate such a data set.

Even if the LOFAR project produces a "perfect" map of the sky, "free" of the corrupting influences of the ionosphere and interference, signals from the astronomical sources located between the LOFAR telescope and the sources of signal from the epoch of reionisation need to be dealt with. The most obvious contaminating sources are the foreground discrete Galactic and extra-Galactic radio sources, which include radio galaxies, quasars, starburst galaxies, and cluster halos. There are two ways of taking their influence into account: (i) all these sources are synchrotron emitters, which have very smooth radio spectra, in principle relatively easily to be distinguished from the sharp $21 \mathrm{~cm}$ spectral features; (ii) the EOR signal will mainly be captured using the smaller few-km size baselines. The longer baselines can then be used to image the discrete sources and subtract them from the smaller baselines. Such a subtraction technique has for example been employed in earlier experiments to detect the spatially extended Sunyaev-Zeldovich effect produced by extended hot gas in galaxy clusters amidst unresolved discrete sources (e.g. Jones et al. 1993). A second and probably much more challenging contaminant is the diffuse polarised emission. This is probably due to Faraday rotation induced by the local ISM confined at distances from us of up to a few 100 pc (Haverkorn et al. 2004). Maps of this emission show clearly angular structure on scales relevant for the EOR experiment. Its spectral behaviour is however probably smooth enough for it to be distinguished from the EOR signals. 
The level of contamination of all these sources and ways to take this into account during the analysis of the EOR signal has been discussed by a number of authors in much greater detail then we could do here. For more details, we refer to: Shaver et al. (1999), Santos et al. (2005), de Bruyn et al. (2005), and reference therein.

After dealing with all these technical issues, the statistical analysis of the resulting 3 -D maps should result in a spectrum of the $21 \mathrm{~cm}$ induced brightness fluctuations as a function of angular scale and redshift/frequency. Different reionisation histories are predicted to produce different fluctuation spectra (e.g. Furlanetta et al. 2004). This will be used to address what is probably the most important question: what class of object is providing the ionising photons: Population III stars, (mini)-quasars, globular clusters, or (mini-halos)? For example, Silk \& Zaroubi (2005) suggested that the steepness of the rise of a reionisation signature would be a powerful discriminator between ionisation by stars or quasars.

Another interesting way to study the epoch of reionisation is to use radio sources located at the epoch of reionisation as background sources against which to observe $21 \mathrm{~cm}$ absorption (e.g. Carilli et al. 2002). Different signatures include: (i) 1\% semi-continuum absorption by the neutral IGM; (ii) few $\mathrm{km} / \mathrm{s}$ narrow absorption lines due to structure that will later form the Ly $\alpha$ forest; and 10 - $100 \mathrm{~km}$ broader lines due absorption by (iii) mini-halos and (iv) protogalaxies. The feasibility of these studies critically depends on the number density and brightness of the radio sources during the epoch of reionisation. On the basis of luminosity functions for distant radio galaxies from Jarvis et al. (2001), Kanekar \& Briggs (2004) show that there is a good prospect that LOFAR can carry out such absorption studies.

\section{References}

Carilli, C. L., Gnedin, N. Y., Owen, F., 2002, ApJ, 577, 22

Cen, R., 2003, ApJ, 591, L5

Fan, X., Strauss, M. A., Schneider, D. P., Becker, R. H., White, R. L., Haiman, Z., Gregg, M., Pentericci, L., Grebel, E. K., Narayanan, V. K., Loh, Y., Richards, G. T., Gunn, J. E., Lupton, R. H., Knapp, G. R., Ivezić, Ž., Brandt, W. N., Collinge, M., Hao, L., Harbeck, D., Prada, F., Schaye, J., Strateva, I., Zakamska, N., Anderson, S., Brinkmann, J., Bahcall, N. A., Lamb, D. Q., Okamura, S., Szalay, A., York, D. G., 2003, AJ, 125, 1649

Furlanetto, S. R., Zaldarriaga, M., Hernquist, L., 2004, ApJ, 613, 16

Harwit, M., 1981, Cosmic discovery. The search, scope, and heritage of astronomy, Brighton: Harvester Press, 1981

Haverkorn, M., Katgert, P., de Bruyn, A. G., 2004, A\&A, 427, 549

Jarvis, M. J., Rawlings, S., Willott, C. J., Blundell, K. M., Eales, S., Lacy, M., 2001, MNRAS, 327,907

Jones, M., Saunders, R., Alexander, P., Birkinshaw, M., Dilon, N., Grainge, K., Hancock, S., Lasenby, A., Lefebvre, D., Pooley, G., 1993, Nature, 365, 320

Kanekar, N., Briggs, F. H., 2004, New Astr. Rev., 48, 1259

Kogut, A., Spergel, D. N., Barnes, C., Bennett, C. L., Halpern, M., Hinshaw, G., Jarosik, N., Limon, M., Meyer, S. S., Page, L., Tucker, G. S., Wollack, E., Wright, E. L., 2003, ApJS, 148,161

Noordam, J. E., 2004, Proc. SPIE, 5489, 817

Santos, M. G., Cooray, A., Knox L., 2004, ArXiv astrophysics e-prints

Shaver, P. A., Windhorst, R. A., Madau, P., de Bruyn, A. G., 1999, A\&A, 345, 380

White, R. L., Becker, R. H., Fan, X., Strauss, M. A., 2003, AJ, 126, 1

Zaroubi, S., Silk, J., 2005, MNRAS, p. L40+ 